\title{
Mental health services in China
}

\author{
MICHAEL R. PHILLIPS
}

Neuropsychiatric conditions in China pose a huge and growing public health problem that is unrecognized by policy makers, the health care establishment, and the public. The Global Burden of Disease project (Murray \& Lopez, 1996a, b) estimates that in 1990 over $5 \%$ of the Chinese population (66 million individuals) suffered from mental disorders and that by 2020 neuropsychiatric conditions and suicide will account for $15.0 \%$ of the total burden of illness for men and $26.8 \%$ of the total burden of illness for women. The most important problems are depression, suicide, bipolar disorder, schizophrenia, obsessive-compulsive disorder, dementia, and alcohol abuse; in 1998 depression was the second most important health problem in the country (accounting for $6.9 \%$ of all DALYs lost), and suicide was the fourth most important problem (accounting for $4.2 \%$ of all DALYs lost) (WHO, 1999).

Like in other resource-poor countries, most persons in China suffering from neuropsychiatric conditions do not receive necessary treatment. The Global Burden of Disease study estimates that in 1990 only $5 \%$ of persons suffering from depression or bipolar disorder received treatment (versus $35 \%$ in developed countries) and only $30 \%$ of persons suffering from schizophrenia received treatment (versus $80 \%$ in developed countries). Moreover, China's recent economic reforms have dramatically altered the provision of health care and led to decreased comprehensiveness and accessibility of mental health services. New initiatives are urgently needed to increase awareness of the importance of neuropsychiatric problems to overall health and to mobilize the political will needed to undertake major reforms in the structure and financing of mental health services.

Indirizzo per la corrispondenza: Professor M.R. Phillips, Beijing Hui Long Guan Hospital, Research Center of Clinical Epidemiology, Beijing 100096 (People's Republic of China).

Fax: +86-10-6271.2471

E-mail: phillips@public3.bta.net.cn

\section{INPATIENT SERVICES}

After the founding of the People's Republic of China in 1949, there was no entity responsible for the overall coordination of social welfare services, so each government ministry developed services for its own constituency. Several ministries developed mental health services (primarily psychiatric hospitals): the Ministry of Health developed services for the general public, the Ministry of Civil Affairs for Veterans and for the destitute, the Ministry of Public Security for mentally ill offenders, the Ministry of Defense for active servicemen, the Ministry of Railways for railway workers, and so forth. Within each ministry the administration of a particular facility could be at the national, provincial, municipal, county, or even enterprise level.

Lacking a central coordinating agency for mental health services, the precise number of facilities is unknown, but it is possible to make a reasonable estimate. As of 1995 the Ministry of Health operated 482 specialized psychiatric hospitals, the Ministry of Civil Affairs operated about 200 psychiatric hospitals, about 85 psychiatric hospitals were operated by other ministries, and about 150 small psychiatric hospitals were collectively or privately owned (Phillips, 1998). The total number of psychiatric beds in these hospitals was about $141,000,88 \%$ of which are in hospitals operated by the Ministries of Health and Civil Affairs. Over $85 \%$ of the inpatients at these hospitals suffer from schizophrenia or other psychotic disorders. Almost all of these psychiatric hospitals are in urban or suburban settings. The heaviest concentration is in large cities such as Shanghai and Beijing; in Shanghai there are 6.5 psychiatric beds per 10,000 population, more than five-fold the national average of 1.2 beds per 10,000 population.

At least $90 \%$ of all mental health services available in China are provided by these specialty psychiatric hospitals. With very few exceptions, there are no psychiatric wards in general hospitals and primary care health 
workers do not provide basic mental health services. With the exception of a few experimental programs and the rural counties of the large metropolitan districts (e.g., Shanghai and Beijing), the vast majority of the rural population - $70 \%$ of the total population - has no access to mental health services or psychiatric medications, so family members in rural areas must bring mentally ill individuals to the nearest city for psychiatric treatment.

Initially these hospitals were directly supported by government revenues, so the costs to consumers were relatively low, and patients without health insurance (over $80 \%$ of the population) had charges reduced by $50 \%$. But during the economic reform era (which started in 1978) hospitals have been expected to become increasingly self-sufficient, so they have had to cover their operating expenses by increasing patient revenues. Over the last 15 years the relative cost of inpatient care to consumers has risen dramatically, more than twice as fast as the increase in incomes (Phillips et al., 1997). Moreover, hospitals no longer reduce the charges for uninsured patients, so many patients have difficulty affording inpatient care.

As financial support from central authorities dwindles, many hospitals have tried to increase their income by providing fee-for-service care to persons outside their original mandate. There is now intense competition between the different types of hospitals for the limited number of fee-paying patients. In some areas where there are no inpatient services or where the available services are quite expensive, local government bodies below the county level and private individuals have opened small for-profit hospitals (usually of poor quality) that compete for patients with the larger hospitals run by the various ministries. Low occupancy rates and falling revenues have forced many psychiatric hospitals to close wards, and some hospitals have shut down.

\section{OUTPATIENT AND COMMUNITY BASED SERVICES}

Almost all outpatient psychiatric services are provided in the outpatient departments of psychiatric hospitals; there are very few freestanding community psychiatric clinics, and general hospitals do not have psychiatric outpatient clinics. Prior to the mid-1980s most psychiatric hospitals also provided extensive 'homebed' services, sending professional staff to the homes of severely ill patients to help avert hospitalization. But the need to become economically self-sufficient has forced hospitals to cut back on cost-effective community services that are not profitable for the hospital or that reduce hospital occupancy rates, such as home-bed services and family-based interventions. The current method of reimbursement for health services employed by medical insurance firms reinforces this emphasis on inpatient services.

Other community based services for the mentally ill - such as guardian networks and sheltered workshops for the rehabilitation of the chronically mentally ill - are only available in some of the large urban centers (Zhang $e t$ al., 1994), and in most of these locations the long-term financial viability of these services is doubtful. In the new economic environment, sheltered workshops are rarely able to achieve the goal of returning patients to regular work. Industries can no longer depend on government subsidies to cover operating loses, so employers are extremely reluctant to assume the potential welfare burden of hiring persons with a history of mental illness. In the urban areas there are now large numbers of expatients with normal or near normal work function who remain at home with nothing to do.

The Work Programme for Disabled Persons During the 9th five-year Development Plan (1996-2000), that is supported by the All China Disabled Persons Federation (a quasi-governmental organization lead by Deng Pufang, Deng Xiaoping's disabled son), includes a plan to provide basic community mental health services to 200 urban and rural sites around the country. The success of the plan has depended on local funding and on local administrative and professional support; these types of support are often insufficient, so it is unlikely that the plan's ambitious goals will be achieved. Moreover, the services envisaged in the plan are for the chronically mentally ill (i.e., those with a "psychiatric disability"), not for persons with depression or other less serious conditions. There are no community based screening programs for depression anywhere in the country, and only a few locations have community based suicide prevention programs.

Awareness of the importance of psychological problems is changing in urban areas (and, to a much lesser extent, in rural areas). This increased awareness has lead to public demands for new types of services: counselling for minor psychiatric problems, telephone hotlines for crisis counselling, community based geriatric services for the rapidly increasing numbers of elderly patients with Alzheimer's dementia, and psychological services for children (partly because of concerns about the psychological effects of single-children families). China's formal mental health services are ill equipped to meet these demands, largely because they are based 
in specialty psychiatric hospitals (many distressed individuals are unwilling to attend outpatient clinics situated in psychiatric hospitals) and partly because mental health professionals have no formal training in dealing with these types of problems.

With few exceptions, the limited services that have evolved in urban areas to deal with these new types of psychological problems are not part of the mental health care system and have no professional mental health providers or consultants. 'Counselling' clinics have opened in schools, in street-level clinics, and in general hospitals (the most advanced, 'third-level' general hospitals are required to provide counselling services). Even the hospital-based counselling services in general hospitals rarely have mental health staff; they are usually staffed by neurologists and general medical nurses whom have no training in mental health and little or no training in dealing with psychological issues. Telephone hotlines for different types of psychological problems have sprung up in China's large urban centers over the last decade (Shanghai has 20 active hotlines); they are popular because of they are free of charge and because users can seek help anonymously, but most of them are staffed by volunteers with little or no training.

\section{MENTAL HEALTH PROVIDERS}

The personnel available to provide mental health services are largely limited to psychiatrists and psychiatric nurses, almost all of who work in psychiatric hospitals. As part of the economic reforms medical and nursing graduates are being given more choice about where they go after graduation (previously they were assigned by the state); few are willing to be assigned to psychiatry because of its low status within medicine and because of the stigma associated with the mentally ill and, by association, with those who have frequent contact with the mentally ill. Moreover, many current psychiatric staff are leaving because the financial crisis at psychiatric hospitals (largely due to low occupancy rates) results in much lower incomes than for staff in other medical specialties. In 1991 psychiatric beds represented about $5 \%$ of the total number of hospital beds in the country, but only $1 \%(11,570)$ of the 1.01 million graduates of 5-year medical schools in the country worked as psychiatrists (Editorial Committee of the China Health Annual, 1992).

The range of services provided is quite limited. In most locations medical graduates who are assigned to psychiatric hospitals are given little or no formal trai- ning in psychotherapy, substance abuse, or in the management of mental illnesses in children or in the elderly; their training is focused on the psychopharmacological treatment of schizophrenia and other psychoses. Psychiatric nurses' role is confined to that of "guardian" on inpatient wards; other than administer medications they rarely assume any therapeutic role. There are no psychiatric social workers or occupational therapists and few clinical psychologists, so community outreach and professional counselling are rarely provided.

There are no where near enough health professionals trained to provide the level of community mental health services needed. Health providers in general medical hospitals and clinics receive almost no mental health training as part of their basic medical education, so they are unable (and often unwilling) to provide basic mental health services as part of their routine clinical practice. Psychiatrists and psychiatric nurses are, moreover, reluctant to work outside of hospitals because the job security and salaries provided in the community are much less than those for inpatient work.

\section{THE UTILIZATION OF SERVICES}

Both by tradition and by law families in China are responsible for the care and supervision of seriously ill family members. Well over $90 \%$ of individuals with serious mental disorders in China live with family members, compared to about $40 \%$ in the United States (Phillips, 1993). The family, not the individual, makes most of the decisions about when and where the individual seeks treatment and about whether or not the individual will be hospitalized.

Family members of individuals suffering from depression, obsessive compulsive disorder, and other important non-psychotic mental disorders do not usually recognize the symptoms as mental problems. Many do not seek any treatment for these problems, but if they do seek treatment, they usually first seek treatment from general physicians. Lacking training in the recognition and management of mental problems, general physicians often misdiagnosed these conditions as physical disorders, so many patients miss the opportunity to receive effective treatment. Even if the psychological nature of the problem is recognized by the patient, the family, and the general doctor, most patients prefer to suffer on their own rather than seek psychiatric help and run the risk of being stigmatized as "mentally ill". Similarly, families in which a family member develops a serious psychological problem such as schizophrenia try 
to manage the problem at home for as long as possible to prevent neighbours and others discovering the secret; only as a last resort will families seek help from the formal mental health services.

Serious mental illness can often impoverish a family. The average three-month psychiatric hospitalization for schizophrenia or other psychotic conditions now costs 6,000-8,000 Renminbi (\$725-\$969 \$US) which is more than the mean per capita urban income $(4,377$ Renminbi per year in 1996) and more than twice the mean per capita rural income. This is a heavy financial burden for families of patients without health insurance (the majority) and for the increasing number of patients who have work-based insurance but can not collect benefits because the work unit is in financial difficulties. Many families without insurance will pay for the first hospitalization (often going into debt to do so) on the hope that inpatient treatment is curative; when the patient relapses families are reluctant or unable to make the financial sacrifice a second time, so they try to manage the acutely disturbed patient in the home. One solution would be to shorten the average length of hospitalization, but hospitals are reluctant to do this because it would decrease revenues, and there are no community services available to provide the intensive level of post-hospitalization care necessary after a brief hospitalization.

\section{COORDINATION OF MENTAL HEALTH SERVICES}

It is extremely difficult to ensure the quality and universal availability of mental health services. The sources of funding, administrative structure, access to medical graduates and other trained personnel, and mandated clinical standards (if any) of the different types of hospitals and community services vary tremendously. Even within a single ministry it is difficult to coordinate services that are administered by departments at different administrative levels (i.e., provincial, municipal, county, and township). There is no single department that manages mental health services within the Ministry of Health (or in the other ministries that provide mental health services); and the economic reforms have, in any case, led to a decentralization of decisionmaking powers, so central authorities no longer have the power to control the provision of services at the local level. There are few legal means to regulate the rapidly developing private sector, and the new psychological counselling clinics are often unwilling to be con- sidered part of the mental health network. Hospitals are reluctant to provide high-quality community based services that would reduce their occupancy rates (and, thus, revenue). Perhaps most importantly, the current overriding concern of all institutions is for financial profitability (or survival), so the intense competition for patients between the different types of psychiatric hospitals makes it difficult to coordinate the development of services.

The First National Congress on Mental Health (held in Nanjing in 1958) attempted to improve the cross-ministry coordination of mental health services by recommending the formation of local and regional mental health coordinating committees that included cadres from the Ministries of Health, Civil Affairs, and Public Security (the so-called "three-man leading groups"). Most large cities established these committees, but the majority of them have stopped functioning or become ineffective over the last 15 years (Phillips \& Pearson, 1994). One of the regulations in a 1992 health law recommended the re-establishment of these leadership groups (which now includes a representative from the All China Disabled Persons Federation) at the national, provincial, urban, and county levels throughout the country. With the exception of the large urban areas, few locations have established these groups; and the mental health leadership groups that are in existence have limited power and resources to affect the development of services.

\section{PROSPECTS FOR THE FUTURE}

Focussed on high-cost inpatient services for psychotic patients, China's mental health care system is neither addressing the current problems of depression and suicide nor preparing to address the looming problems of substance abuse and senile dementia. The failure to provide high-quality, comprehensive and affordable mental health services in the community - particularly in rural areas - and the failure to convince non-psychiatric health professionals to provide basic mental health services are seriously undermining attempts to improve the level of mental health of the nation.

Initiating and sustaining the reforms needed to overcome these obstacles will not be easy. Changing attitudes about mentally ill patients and about the importance of psychological health requires a sustained effort by a committed group of activists, sophisticated understanding of the social and psychological factors that influence public attitudes, adequate resources, and the full 
cooperation of the media. Changing the emphasis of mental health care from inpatient settings to outpatient and community settings and training the personnel needed to provide the new types of services requires the longterm coordinated effort of many departments and social agencies, so the active support of regional and local government leaders is absolutely essential.

The recent CHINA/WHO Mental Health Awareness Raising Conference held in Beijing (11-13 November 1999) attempted to mobilize the political will to develop and implement an action plan to address these problems. The proclamation released at the conclusion of the meeting by the eight ministries and other agencies that participated indicated recognition of the magnitude of the problems and the need to fundamentally change the current treatment system. The difficult next step is to convert this proclamation into concrete action.

\section{REFERENCES}

Editorial Committee of the China Health Annual (Ed.) (1992). 1992
Yearbook of Public Health in the People's Republic of China [in Chinese]. People's Health Press: Beijing.

Murray C.J.L. \& Lopez A.D. (1996a). Global Health Statistics. Harvard University Press: Cambridge.

Murray C.J.L. \& Lopez A.D. (1996b). The Global Burden of Disease. Harvard University Press: Cambridge.

Phillips M.R. (1993). Strategies used by Chinese families coping with schizophrenia. In Chinese Families in the Post-Mao Era (ed. D. Davis and S. Harrell), pp. 277-306. University of California Press: Berkeley and Los Angeles.

Phillips M.R. (1998). The transformation of China's mental health services. China Journal 39, 1-36.

Phillips M.R. \& Pearson V. (1994). Future opportunities and challenges for the development of psychiatric rehabilitation in China. British Journal of Psychiatry 165, suppl. 24, 128-142.

Phillips M.R., Lu S.H. \& Wang R.W. (1997). Economic reforms and the acute inpatient care of schizophrenia: the Chinese experience. American Journal of Psychiatry 154, 1228-1234.

World Health Organization (1999). World Health Report 1999. WHO: Geneva.

Zhang M.Y., Yan H.Q. \& Phillips M.R. (1994). Community-based psychiatric rehabilitation in Shanghai: facilities, services, outcome, and culture-specific characteristics. British Journal of Psychiatry 165, suppl. 24, 70-79. 